03

\title{
Пропускание света тонким металлическим экраном с бесконечным массивом периодических нанощелей
}

\author{
(C) Н.В. Селина \\ Кубанский государственный технологический университет, \\ 350000 Краснодар, Россия \\ e-mail: selina_natalia@mail.ru \\ Поступила в редакцию 09.04.2018 г. \\ В окончательной редакции 01.02.2019 г. \\ Принята к публикации 11.06.2019 г.
}

Предложен рациональный способ вычисления пропускания света тонким металлическим экраном с бесконечным периодическим массивом субволновых щелей. Результаты расчета хорошо согласуются с известными экспериментальными и теоретически рассчитанными данными.

Ключевые слова: наноотверстия в тонком металлическом экране; кусочно-однородная среда с плоскопараллельными границами раздела.

DOI: $10.21883 /$ OS.2019.10.48371.101-19

\section{Введение}

Согласно теории Бете, пропускание света с длиной волны $\lambda$ через малое отверстие в тонком металлическом экране описывается формулой $T \sim(a / \lambda)^{4}(a-$ характерный размер апертуры) [1]. При условиях принадлежности длины волны к видимому диапазону и наномасштабе отверстия пропускание света рассматриваемой структурой должно быть малым. Однако в экспериментально наблюдаемых спектрах максимальное пропускание для некоторых резонансных длин волн превышает это значение в десятки и сотни раз. В начале прошлого века Р. Вуд наблюдал аномально слабое отражение света некоторых длин волн видимого диапазона от металлической дифракционной решетки с нанорельефом. Такая особенность названа аномалией Вуда. В 1941 г. У. Фано показал, что этот эффект связан с возбуждением поверхностных плазмонов [2].

В дальнейшем активно проводились исследования прохождения света через металлическую пленку, перфорированную наноотверстиями (нанощелями) [3-7]. Было предпринято множество описаний этого физического процесса. В частности, в работах [4-7] содержатся результаты численного решения уравнений Максвелла, а в [3] приведена аналитическая формула для пропускания света массивом нанощелей в тонкой металлической пленке. Она получена методом „сшивания“ полей на границах разделов сред „металл-диэлектрик“. С помощью этой теории рассчитывается пропускание света металлодиэлектрическими структурами с малым отношением ширины щелей к периоду. В настоящей статье к рассматриваемой задаче применена новая методика расчета кусочно-однородных сред. Экран выбирается тонким (меньшим длины волны), поскольку этого требуют условия поперечного (по отношению к экрану) плазмонного резонанса. Разработанный алгоритм действует в условиях произвольного соотношения материальных и геометрических параметров периодической структуры, расчетные формулы упрощены в сравнении с предложенными в [3], метод легко обобщается на любое число границ раздела сред. Массив из нескольких экранов с субволновыми щелями исследован непосредственным численным решением уравнений Максвелла.

\section{Теоретическое рассмотрение задачи}

Рассмотрим распространение монохроматической электромагнитной волны в среде, однородной вдоль некоторой выделенной оси, обозначим ее $O y$. Выберем систему координат таким образом, чтобы волна распространялась в плоскости $x O z$. Избранные геометрические параметры обусловливают независимость всех величин, характеризующих падающую и проходящую через структуру волну, от координаты $y$. Закон распространения электромагнитной волны описывается волновым уравнением, которое выводится из уравнений Максвелла для напряженностей электрического и магнитного полей в волне [5]:

$$
\Delta \mathbf{H}+\frac{1}{\varepsilon}[\operatorname{grad} \varepsilon \times \operatorname{rot} \mathbf{H}]+\frac{\varepsilon \omega^{2}}{c^{2}} \mathbf{H}=0,
$$

$\omega$ - частота электромагнитной волны, $\varepsilon$ диэлектрическая проницаемость среды:

$$
\varepsilon(x, z)=\varepsilon_{1}+\left(\varepsilon_{2}-\varepsilon_{1}\right) T(x, z),
$$

$T(x, z)$ - ступенчатая функция, принимающая значение, равное 0 в области 1 с диэлектрической проницаемостью $\varepsilon_{1}$ и границами с некоторыми произвольными координатами, определенными для конкретной задачи, и значение 1 вне области 1 , которую 
обозначим как область 2 :

$$
T(x, y)=\left\{\begin{array}{l}
1, \varepsilon(x, z)=\varepsilon_{2}, \\
0, \varepsilon(x, z)=\varepsilon_{1},
\end{array}\right.
$$

$\varepsilon_{1}, \varepsilon_{2}-$ диэлектрические проницаемости областей 1 и 2 соответственно. Для дальнейших расчетов введем волновое число электромагнитной волны в вакууме $k_{0}$ :

$$
\frac{\omega^{2}}{c^{2}}=k_{0}^{2}
$$

Поскольку описывается явление плазмонного резонанса, рассмотрим волну ТМ-поляризации, у которой волновой вектор лежит в плоскости $x O z$. При таком выборе координат отлична от нуля только $y$-компонента напряженности магнитного поля. Учитывая, что диэлектрическая проницаемость меняется в направления $x$ и $z$, из (1) получаем

$$
\frac{\partial^{2} H_{y}}{\partial x^{2}}-\frac{1}{\varepsilon} \frac{\partial \varepsilon}{\partial x} \frac{\partial H_{y}}{\partial x}+\frac{\partial^{2} H_{y}}{\partial z^{2}}-\frac{1}{\varepsilon} \frac{\partial \varepsilon}{\partial z} \frac{\partial H_{y}}{\partial z}=-H_{y} \varepsilon k_{0}^{2} .
$$

Решение этой задачи для волны ТЕ-поляризации аналогично.

Для решения задачи кусочно-однородной среды с двумя возможными значениями диэлектрической проницаемости запишем квадрат волнового вектора в виде двух произвольных частей:

$$
\varepsilon_{i} k_{0}^{2}=k_{x i}^{2}+k_{z i}^{2}, \quad i=1,2 .
$$

Умножая уравнение (3) на $\frac{\varepsilon_{1}}{\varepsilon}$, а также прибавляя и вычитая из него выражения $\frac{\varepsilon}{\varepsilon_{1}}\left(\frac{\varepsilon_{1}}{\varepsilon H} \frac{\partial H_{y}}{\partial x}\right)^{2}$ и $\frac{\varepsilon}{\varepsilon_{1}}\left(\frac{\varepsilon_{1}}{\varepsilon H} \frac{\partial H_{y}}{\partial z}\right)^{2}$ в левой части уравнения, приводим полученный результат к виду

$$
\begin{aligned}
& \frac{\partial}{\partial x}\left(\frac{\varepsilon_{1}}{\varepsilon H_{y}} \frac{\partial H_{y}}{\partial x}\right)+\frac{\varepsilon}{\varepsilon_{1}}\left(\frac{\varepsilon_{1}}{\varepsilon H_{y}} \frac{\partial H_{y}}{\partial x}\right)^{2} \\
& +\frac{\partial}{\partial z}\left(\frac{\varepsilon_{1}}{\varepsilon H_{y}} \frac{\partial H_{y}}{\partial z}\right)+\frac{\varepsilon}{\varepsilon_{1}}\left(\frac{\varepsilon_{1}}{\varepsilon H_{y}} \frac{\partial H_{y}}{\partial z}\right)^{2}=-\varepsilon_{1} k_{0}^{2} .
\end{aligned}
$$

Такая группировка сомножителей в членах уравнения (4) позволяет ввести для всех областей кусочно-однородной среды функции фазы $a$ по направлению $x$, и фазы $b$ по направлению $z$ согласно равенствам

$$
\begin{aligned}
\frac{1}{H_{y}} \frac{\partial H_{y}}{\partial x} & =-\frac{\varepsilon}{\varepsilon_{1}} k_{x 1} \operatorname{tg}(a), \\
\frac{1}{H_{y}} \frac{\partial H_{y}}{\partial z} & =-\frac{\varepsilon}{\varepsilon_{1}} k_{z 1} \operatorname{tg}(b) .
\end{aligned}
$$

Учитывая (5) и (6), преобразуем уравнение (4) к виду

$$
\begin{aligned}
& k_{x 1} \frac{\partial \operatorname{tg}(a)}{\partial x}-\frac{\varepsilon}{\varepsilon_{1}}\left(\left(k_{x 1} \operatorname{tg}(a)\right)^{2}+k_{x 1}^{2}\right. \\
& \left.+T(x, z)\left(k_{x 2}^{2} \frac{\varepsilon_{1}^{2}}{\varepsilon_{2}^{2}}-k_{x 1}^{2}\right)\right)+k_{z 1} \frac{\partial \operatorname{tg}(b)}{\partial z} \\
& -\frac{\varepsilon}{\varepsilon_{1}}\left(\left(k_{z 1} \operatorname{tg}(b)\right)^{2}+k_{z 1}^{2}+T(x, z)\left(k_{z 2}^{2} \frac{\varepsilon_{1}^{2}}{\varepsilon_{2}^{2}}-k_{z 1}^{2}\right)\right)=0 .
\end{aligned}
$$

По аналогии с традиционным решением положим, что функция $a(x, z)$ зависит непрерывно от координаты $x$ и через функцию Хэвисайда (через кусочно-непрерывную диэлектрическую проницаемость) от координаты $z$, а $b(x, z)$ - наоборот. Тогда последнее уравнение разбивается на два: равенство нулю первых пяти слагаемых и равенство нулю оставшихся слагаемых. Из этих уравнений следует, что в области, заполненной средой 1 ,

$$
\frac{\partial a(x, z)}{\partial x}=k_{x 1}, \quad \frac{\partial b(x, z)}{\partial z}=k_{z 1} .
$$

Для них решение уравнения (3) находится интегрированием (5), (6) и имеет вид

$$
H_{y}(x, z)=H_{0} \cos (a) \cos (b) .
$$

Вводя обозначения

$$
\begin{aligned}
& \frac{1}{\alpha^{2}}=\frac{k_{x 2}^{2}}{k_{x 1}^{2}} \frac{\varepsilon_{1}^{2}}{\varepsilon_{2}^{2}}, \quad \frac{1}{\beta^{2}}=\frac{k_{z 2}^{2}}{k_{z 1}^{2}} \frac{\varepsilon_{1}^{2}}{\varepsilon_{2}^{2}}, \\
& \tilde{a}(x, z)=\operatorname{arctg}(\alpha \operatorname{tg} a(x, z))), \\
& \tilde{b}(x, z)=\operatorname{arctg}(\beta \operatorname{tg} b(x, z))),
\end{aligned}
$$

запишем уравнения (7) для области, заполненной второй средой:

$$
\frac{\partial \tilde{a}(x, z)}{\partial x}=k_{x 2}, \quad \frac{\partial \tilde{b}(x, z)}{\partial z}=k_{z 2} .
$$

Тогда формулы (5) и (6) примут вид

$$
\frac{1}{H_{y}} \frac{\partial H_{y}}{\partial x}=-k_{x 2} \operatorname{tg}(\tilde{a}), \quad \frac{1}{H_{y}} \frac{\partial H_{y}}{\partial z}=-k_{x 2} \operatorname{tg}(\tilde{b}) .
$$

Интегрируя эти равенства с учетом (8), находим решение для областей, заполненных средой 2 :

$$
H_{y}(x, z)=H_{0} \cos (\tilde{a}) \cos (\tilde{b}) .
$$

Решение определено с точностью до постоянного слагаемого в функциях фазы. Определим эти константы в каждой из областей. В области, содержащей начало координат, в положительном направлении оси $O X$ от этой точки, функция $a(x, z)$ равна

$$
a(x)=k_{x 1} x .
$$

Здесь положили, что рассматриваемая область заполнена средой 1 . Положим также, что ее протяженность по оси $X$ равна $d$, тогда в следующей области аргумент косинуса имеет вид

$$
\tilde{a}(x)=k_{x 2}(x-d)+\operatorname{arctg}\left(\alpha \operatorname{tg}\left(k_{x 1} d\right)\right) .
$$

Аналогично можно записать решение для любого числа областей, заполненных разными средами. Так для направления оси $Z$ имеем

$$
\tilde{b}(z)=k_{z 2}(z-h)+\operatorname{arctg}\left(\beta \operatorname{tg}\left(k_{z 1} h\right)\right) .
$$


Здесь $h$ - протяженность области, содержащей начало координат, по направлению оси $Z$.

Рассматриваемые функции, следовательно, и зависящая от них напряженность магнитного поля волны являются непрерывными на границах раздела сред в направлениях $x$ и $z$. Кроме того, в таком представлении решения непрерывна и тангенциальная (для таких границ раздела сред) компонента напряженности электрического поля волны, поскольку производные функции напряженности магнитного поля волны по координатам $x$ и $z$ определяются следующими формулами:

$$
\begin{aligned}
& \frac{\partial H_{y}}{\partial x}=-\frac{\varepsilon}{\varepsilon_{1}} k_{x 1} \operatorname{tg}(a) H_{y}, \\
& \frac{\partial H_{y}}{\partial z}=-\frac{\varepsilon}{\varepsilon_{1}} k_{z 1} \operatorname{tg}(b) H_{y} .
\end{aligned}
$$

Таким образом, полученное решение уравнения (3) по сути совпадает с традиционным, поскольку включает в себя функции косинуса от координат, умноженных на волновой вектор в среде. Найденное решение лишь определяет константу, прибавляемую к значению, называемому обычно фазой волны. Преимущество данного решения состоит в том, что определяющая его функция непрерывна на границе сред и записывается сразу для всех сред-компонентов.

Далее рассмотрим структуру с двумя границами раздела сред в направлении оси $O Z$ и бесконечным периодическим массивом щелей в плоскости этих границ (рис. 1), и применяя выведенные формулы, найдем соотношение между материальными и геометрическими характеристиками структуры и оптическими параметрами ее собственной моды - дисперсионное соотношение. Для того, чтобы свет от щелей распространялся в фазе, необходимо положить разность фаз между серединами щелей кратной $2 \pi$ :

$$
\begin{gathered}
a=\frac{k_{x 2} d_{1}}{2}+\operatorname{arctg}\left(\frac{k_{x 1} \varepsilon_{2}}{k_{x 2} \varepsilon_{1}} \operatorname{tg}\left(\frac{k_{x 1} d}{2}+\pi m\right)\right)=\pi n, \\
n, m=1,2 \ldots,
\end{gathered}
$$

где $d-$ ширина щели, $p=d_{1}+d-$ период структуры.

Преобразуя это уравнение, получаем дисперсионное соотношение для волноводной структуры щелей:

$$
\frac{k_{x 1} \varepsilon_{m}}{k_{x 2} \varepsilon_{d}} \operatorname{tg}\left(\frac{k_{x 1} d}{2}\right)+\operatorname{tg}\left(\frac{k_{x 2} d_{1}}{2}\right)=0
$$

При больших значениях $d_{1}$ приходим к дисперсионному уравнению для трехслойной среды - тонкой металлической пленки между диэлектрическими полупространствами. В работе [3] решалось именно это уравнение. Как следует из приведенных выше рассуждений, его можно использовать для определения постоянной распространения волноводной моды в субволновых щелях только в пределе большого периода, сравнимого с длиной волны.

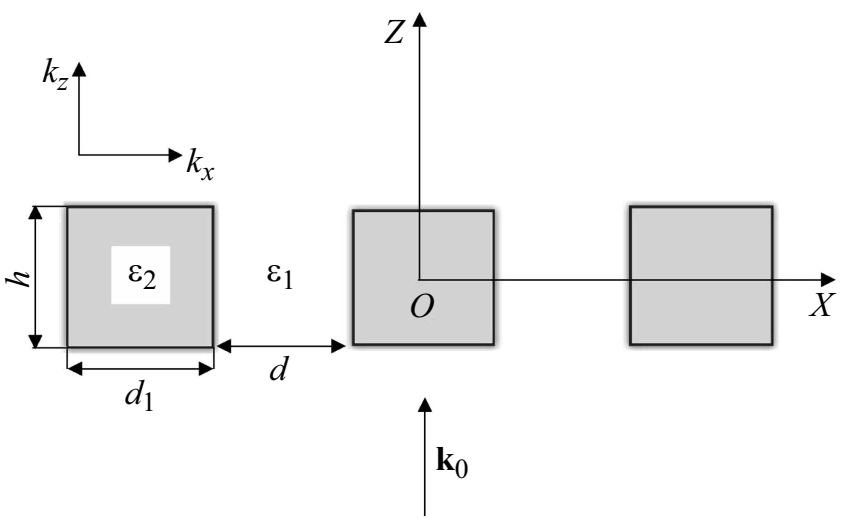

Рис. 1. Схематическое изображение периодической структуры, представляющей собой тонкий металлический экран с бесконечным массивом вертикальных нанощелей: $k_{0}=2 \pi / \lambda$, $\lambda$ - длина волны излучения, $\mathbf{k}_{0}=\mathbf{e}_{z} k_{0}, \mathbf{e}_{z}-$ единичный вектор в направлении оси $O Z$.

Согласно теореме Флоке-Блоха, решение волнового уравнения для периодической структуры есть сумма волн различных порядков дифракции. Определим одну из них. Запишем формулу (9) для волны $n$-го порядка дифракции на границах структуры, находящихся на расстоянии $h / 2$ в обе стороны от координатной плоскости, перпендикулярной оси $O Z$ и разделяющей экран на две равные половины:

$$
\begin{gathered}
H_{n}\left(x_{1}, \frac{h}{2}\right)=H_{n 0} \cos \left(\tilde{a}_{n}\right) \cos \left(\tilde{b}_{n}\right), \\
\tilde{b}_{n}=\operatorname{arctg}\left(\sqrt{\gamma_{n}} \operatorname{tg}\left(\frac{\beta h}{2}\right)\right), \\
\gamma_{n d}=\frac{\beta^{2}}{k_{0}^{2}-k_{x, n}^{2}}, \quad \gamma_{n m}=\frac{\frac{\beta^{2}}{\mathcal{\varepsilon}^{2}}}{k_{0}^{2}-k_{x, n}^{2}} .
\end{gathered}
$$

Индекс $d$ относится к областям, заполненным диэлектриком (среда 1$), m$ - металлом (среда 2).

$$
\alpha=\frac{\varepsilon^{2}\left(\sqrt{k_{0}^{2}-\beta^{2}}\right)^{2}}{\left(\sqrt{\varepsilon k_{0}^{2}-\beta^{2}}\right)^{2}}, k_{x n}=\frac{2 \pi n}{p} .
$$

$\beta$ - постоянная распространения света в щели, $\varepsilon=\varepsilon_{2}$ - диэлектрическая проницаемость металла, $n$ - порядок дифракции. Рассматриваем металлический экран из золота в воздухе $\left(\varepsilon_{1}=1\right)$.

В рассматриваемой структуре по границе вдоль оси $O X$ существуют решения с постоянной $k_{x n}$ и поперечными волновыми векторами: $k_{z n}=\sqrt{k_{0}^{2}-k_{x n}^{2}}$, направленными в обе стороны от координатной плоскости, которые характеризуют рассеяние света посредством дифракции на щелях. Они определяют потоки энергии на границах структуры вместе с волновым вектором волноводной моды щелей. Чтобы определить эти потоки, 
необходимо использовать выражение для напряженности электрического поля.

С этой целью запишем выражение для одной из компонент ( $x$-компонента) электрического поля линейно поляризованной волны, которая определяется производной от магнитного поля по продольной координате:

$$
E(x, z)=i H_{n 0} \cos \left(\tilde{a}_{n}\right) \sin \left(\tilde{b}_{n}\right) .
$$

Для $z$-составляющей вектора Умова-Пойтинга на границах экрана получаем равенство

$$
E H=\operatorname{const}\left(k_{z n}\right) \sqrt{\gamma_{n}} \operatorname{tg}\left(\frac{k_{z n} h}{2}\right)\left(H_{n}(x, z)\right)^{2} .
$$

Таким образом, потоки энергии через границы экрана, перпендикулярные оси $O Z$, переносимые волнами $n$-го дифракционного порядка, равны

$$
P_{1}=-P_{2}=\operatorname{const}\left(k_{z n}\right) \sqrt{\gamma_{n m}} \operatorname{tg}\left(\frac{k_{z n} h}{2}\right)\left(H_{n}\left(x, \frac{h}{2}\right)\right)^{2} .
$$

Далее рассмотрим потоки энергии, переносимые волноводной модой щелей между границами экрана, перпендикулярными оси $O Z$, в двух половинах экрана, разделенных плоскостью $z=0$ :

$$
P_{3}=P_{4}=\operatorname{const} \beta \sqrt{\gamma_{0}} \operatorname{tg}\left(\frac{\beta h}{2}\right)\left(H_{0}\left(x, \frac{h}{2}\right)\right)^{2} .
$$

В структуре формируются также в двух направлениях оси $Z$ поперечные потоки продольных мод с $z$-компонентой волнового вектора, по модулю равной $\beta$, в металлической и диэлектрической (в щелях) частях экрана:

$$
\begin{aligned}
P_{5} & =-P_{6}=\operatorname{const}\left(d_{1} \beta \sqrt{\gamma_{n m}}+d \beta \sqrt{\gamma_{n d}}\right) \\
& \times \operatorname{tg}\left(\frac{\beta h}{2}\right)\left(H_{0}\left(x, \frac{h}{2}\right)\right)^{2} .
\end{aligned}
$$

Произведение вектора Умова-Пойнтинга потока $P_{1}$ в точке с заданными координатами на элемент объема определяет энергию, вытекающую (втекающую) из этой области пространства, а также поглощающуюся в ней за счет перемещения этой части энергии:

$$
d W_{1}=P_{1} S d z=i \sqrt{\gamma_{n m}} \operatorname{tg}\left(\frac{k_{z n} h}{2}\left(H_{n}\left(x, \frac{h}{2}\right)\right)^{2} k_{z n} d z S,\right.
$$

где $S$ - площадь сечения структуры, поперечного оси $Z$.

Действительно, при мнимом значении волнового вектора $k_{z n}$ выражение (10) совпадает с законом Бугера, определяющим ослабление параллельного монохроматического пучка при распространении его в поглощающей среде:

$$
d J=-k \sigma J d z
$$

В этой формуле $J$ - интенсивность волны, $\sigma$ - сечение поглощения в среде, $z$ - координата, изменяющаяся в направлении распространения волны. Согласно физическому определению, интенсивность - это среднее по времени значение модуля вектора Умова-Пойнтинга. Следовательно, выражение (10) учитывает поглощение энергии в структуре.

Проинтегрируем выражение (10), в качестве пространства интегрирования выберем область экрана, заключенную между плоскостями $z=0$ и $z=-h / 2$ :

$$
\begin{aligned}
\int_{-h / 2}^{0}\left(-P_{1}\right) d z & =-\int_{-h / 2}^{0} k_{z n} \sin \left(\tilde{b}_{n}(z)\right) \cos \left(\tilde{b}_{n}(z)\right) d z \tilde{b}_{n}(z) \\
& =-\operatorname{const}\left(C_{n} \sin \left(\tilde{b}_{n}\left(\frac{h}{2}\right)\right)^{2}\right),
\end{aligned}
$$

$C_{n}$ - постоянная, характеризующая амплитуду нулевой моды. В результате получено значение разности энергии, втекающей в область интегрирования и вытекающей из нее, а также поглощающейся в ней, в единицу времени.

Чтобы изолированно учесть в расчетах каждую из мод, представим себе абстрактную ситуацию: в волноводной моде нет затухания волны, нет возбуждения продольных экрану мод ни одного из дифракционных порядков, соответственно нет отражения от структуры щелей. Тогда поток энергии световой волны, втекающий в щели в плоскости $z=-h / 2$, равен потоку энергии волны, вытекающему из щелей в плоскости $z=h / 2$, и пропускание щелей равно единице.

Учитывая, что задачей является расчет отдельно каждой моды, рассмотрим именно такой пример, но с условием, что одна продольная экрану мода $n$-го дифракционного порядка все же возбуждается, и соответственно существует поглощение в этой моде с утечкой энергии в направленииях, прямом и обратном основному потоку волноводной моды. В этом случае пропускание области структуры, заключенной между плоскостями $z=-h / 2$ и $z=0$, равно отношению разности энергии волны, падающей на экран, и энергии, вытекающей из возбужденной моды и поглощающейся в ней, к энергии волны, падающей на экран:

$$
t_{n}^{(-1 / 2)}=\frac{W_{0}-W_{n}}{W_{0}}=1-\frac{C_{n}}{W_{0}} \sin \left(\tilde{b}_{n}(h / 2)\right)^{2} .
$$

Знак „минус“ в этой формуле показывает направление поперечного волнового вектора моды $n$, противоположное основному потоку. Рассмотрим еще и вторую половину области экрана, заключенную между плоскостями $z=0$ и $z=h / 2$, в условиях предыдущего предположения. Соответственно пропускание такой системы отличается от пропускания системы, рассмотренной ранее, знаком направления потока возбуждаемой моды:

$$
t_{n}^{(1 / 2)}=\frac{W_{0}+W_{n}}{W_{0}}=1+\frac{C_{n}}{W_{0}} \sin \left(\tilde{b}_{n}(h / 2)\right)^{2} .
$$


Полное пропускание структуры равно произведению $t_{n}^{(-1 / 2)}$ и $t_{n}^{(1 / 2)}$ :

$$
t_{n}=1-\frac{C_{n}^{2}}{W_{0}^{2}} \sin \left(\tilde{b}_{n}(h / 2)\right)^{4}
$$

В волноводной моде единственный канал утечки энергии - поглощение, поэтому для моды нулевого порядка получаем выражение:

$$
t_{0}=\exp \left(-2 \operatorname{Im}\left(\tilde{b}_{0}(h / 2)\right)\right) .
$$

Поскольку энергия между модами распределяется пропорционально энергетическому объему мод, то для всех $n$ множитель $C_{n}$ одинаков. Значение $C_{n}$ можно найти из равенства, определяющего закон сохранения энергии: втекающая в структуру энергия равна суммарной энергии, вытекающей из структуры и поглощающейся в ней:

$$
2 \sum_{n=1}^{\infty} C_{n} \sin \left(\tilde{b}_{n}(h / 2)\right)^{2}=2 W_{0} .
$$

Откуда получаем амплитуду напряженности поля n-й моды:

$$
C_{n}=\frac{W_{0}}{\sum_{n=1}^{\infty} \sin \left(\tilde{b}_{n}\left(\frac{h}{2}\right)\right)^{2}} .
$$

Пропускание экрана с возбуждением $n$-й моды соответственно равно

$$
t_{n}=1-\frac{\sin \left(\tilde{b}_{n}\left(\frac{h}{2}\right)\right)^{4}}{\left(\sum_{i=1}^{\infty} \sin \left(\tilde{b}_{i}\left(\frac{h}{2}\right)\right)^{2}\right)^{2}}
$$

Полное пропускание рассматриваемой структуры равно произведению значений $t_{n}$ для каждой моды $n$-го порядка дифракции:

$$
T=\prod_{n=0}^{\infty}\left(1-\frac{\sin \left(\tilde{b}_{n}\left(\frac{h}{2}\right)\right)^{4}}{\left(\sum_{i=1}^{\infty} \sin \left(\tilde{b}_{i}\left(\frac{h}{2}\right)\right)^{2}\right)^{2}}\right) .
$$

Синус в этом выражении можно преобразовать:

$$
\begin{aligned}
& \sin \left(\tilde{b}_{n}\left(\frac{h}{2}\right)\right)^{4}=\frac{\gamma_{n}^{2} \operatorname{tg}\left(\frac{\beta h}{2}\right)^{4}}{\left(1+\gamma_{n} \operatorname{tg}\left(\frac{\beta h}{2}\right)^{2}\right)^{2}}, \\
& \sum_{n=1}^{\infty}\left(\tilde{b}_{n}\left(\frac{h}{2}\right)\right)^{2}=\sum_{n=1}^{\infty} \frac{\gamma_{n} \operatorname{tg}(\beta h / 2)^{2}}{1+\gamma_{n} \operatorname{tg}(\beta h / 2)^{2}} .
\end{aligned}
$$

Из результатов такого преобразования следует, что минимумы в пропускании соответствуют следующим условиям.



Рис. 2. Зависимость пропускания нулевого порядка дифракции структурой, изображенной на рис. 1 со следующими параметрами: материал экрана - золото (диэлектрическая проницаемость определялась по формуле Друде с плазменной частотой $9.1 \mathrm{eV}[8]), d=150 \mathrm{~nm}, h=285 \mathrm{~nm}, p=500 \mathrm{~nm}$.

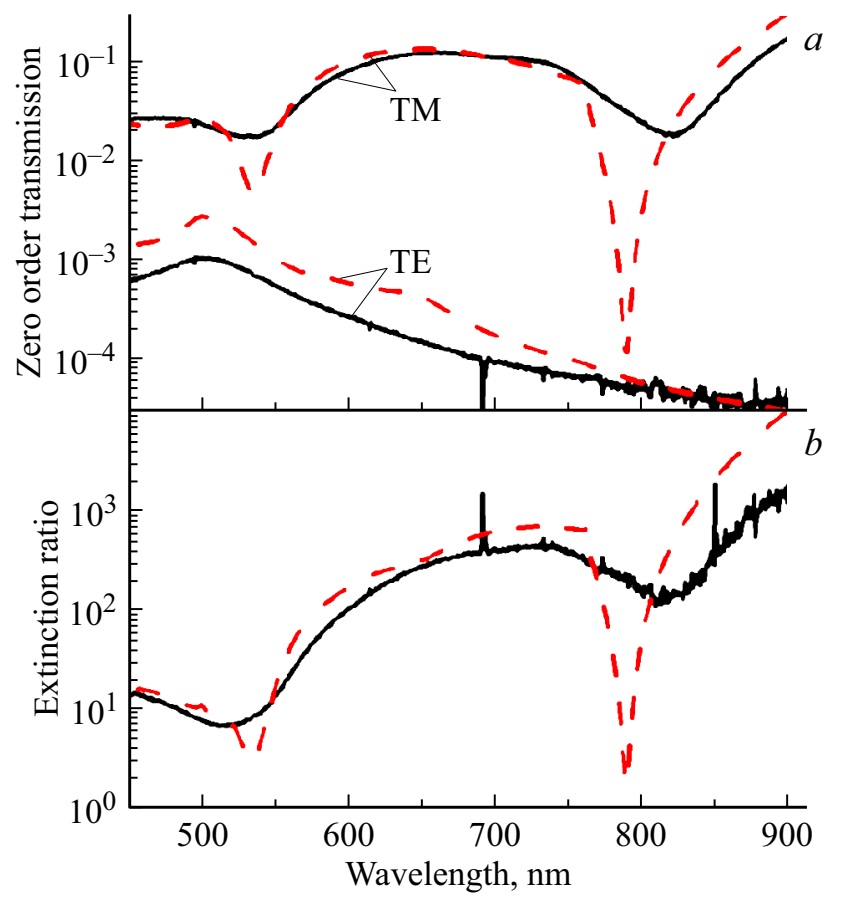

Рис. 3. Спектр пропускания нулевого дифракционного порядка золотого экрана, схематически изображенного на рис. 1, с параметрами $d=150 \mathrm{~nm}, h=285 \mathrm{~nm}, p=500 \mathrm{~nm}$ : сплошная кривая - эксперимент [3], штриховая кривая - настоящая работа.

1. $\gamma_{n} \rightarrow \infty, \lambda \approx \sqrt{\frac{\varepsilon}{\varepsilon+1}} p / n$, случай плазмонного резонанса на внешней границе экрана.

2. $\operatorname{tg}\left(\frac{\beta h}{2}\right) \sqrt{\gamma_{n}}=i$, случай плазмонного резонанса в трехслойной структуре. 
Пропускание моды $n$-го порядка равно пропусканию всей структуры, умноженному на отношение энергетического объема моды к суммарному объему всех мод:

$$
\delta_{n}=T \frac{\sin \left(\tilde{b}_{n}\left(\frac{h}{2}\right)\right)^{2}}{\sum_{i=1}^{\infty} \sin \left(\tilde{b}_{i}\left(\frac{h}{2}\right)\right)^{2}} .
$$

На рис. 2 изображен график зависимости пропускания нулевого порядка дифракции от длины волны излучения, построенный по формулам (11), (12) с использованием значений $\beta$, полученных при решении дисперсионного уравнения для волноводной структуры щелей. На графике пропускания нулевого порядка дифракции должны быть относительно широкие минимумы вблизи значений длины волны $\frac{p}{n}$. Обращаясь к рис. 2, замечаем, что минимумам соответствуют длины волн 500 и $900 \mathrm{~nm}$, что определяется плазмонным резонансом в трехслойной металлодиэлектрической структуре. Вид графика хорошо согласуется с экспериментальными данными [3] и результатами расчетов по формулам, определенным „сшиванием“ решений волнового уравнения для каждой из сред на их границе (рис. 3).

\section{Заключение}

Приведен метод расчета пропускания света тонким металлическим экраном с периодическим массивом нанощелей. Описанный способ расчета является точным и удобным. Результаты численного анализа приведенных формул согласуются с основными экспериментальными и теоретическими данными, приведенными в литературе.

\section{Конфликт интересов}

Автор заявляет, что у нее нет конфликта интересов.

\section{Список литературы}

[1] Bethe H.A. // Phys. Rev. 1944. V. 66. P. 163-182.

[2] Fano U. // J. Opt. Soc. Am. 1941. V. 31. P. 213-222.

[3] Guillaumée M. Optical Transmission Through Sub-Wavelength Slits in Metals: from Theory to Applications. Thèse no 4723 école polytechnique fédérale de Lausanne. 2010.

[4] Irannejad M., Zhang J., Yavuz M., Cu B. // Plasmonics. 2014. V. 9. P. 537-544.

[5] Babicheva V. E., Lozovik Y. // J. Opt. Quant. Electron. 2009. V. 41. P. 299-313.

[6] Fu Y., Li K., Kong F.M. // PIER. 2008. V. 82. P. 109-125.

[7] Moreau A., Lafarge C., Laurent N., Edee K., Granet G.// J. Opt. A. 2007. V. 9. P. 165-169.

[8] Johnson P., Christy R. // Phys. Rev. B. 1972. V. 6. P. 4370. 